\title{
Ribbon polymers in poor solvents: layering transitions in annular and tubular condensates
}

\author{
Y. Y. SuZuKI ${ }^{1,2}$ and D. R. M. WilLIAMs ${ }^{3}$ \\ Institut de Physique Théorique, CEA - IPhT, CNRS, URA 2306 F-91191 Gif-sur-Yvette, France \\ Faculty of Engineering, Takushoku University - Hachioji, Tokyo 193-0985, Japan \\ Research School of Physical Sciences, Australian National University - Canberra, ACT 0200, Australia
}

PACS 36.20.Ey - Conformation (statistics and dynamics)

PACS 64.70.Nd - Structural transitions in nanoscale materials

PACS 87.15.ad - Analytical theories

\begin{abstract}
We study the structures of a ribbon or ladder polymer immersed in poor solvents. The anisotropic bending rigidity coupled with the surface tension leads ribbon polymers to spontaneous formation of highly anisotropic condensates in poor solvents. Unlike ordinary flexible polymers these condensates undergo a number of distinct layering transitions as a function of chain length or solvent quality, and the size of condensates becomes non-monotonic function of chain length. We show that the fluctuations of the condensates are in general small and these condensates are stable.
\end{abstract}

Recently considerable attention has been devoted to the - physics of semiflexible polymers, i.e., chains which have significant bending stiffness. These kinds of polymers exhibit interesting physics in the form of liquid crystalline phases and are of great importance in biology and biophysics. For example, DNA is the most well-known semiflexible polymer. Much of the interest has focused on semiflexible chains with isotropic bending elasticity [1-7]. Macroscopically this corresponds to modeling the chain as a cylinder with a circular cross section. In many cases, however, the cross section is highly anisotropic [8-12].

The anisotropic polymer can be modeled as a ribbon, of length $L$, width $w$, and thickness $t$ assuming $L \gg w>t$ (fig (1) with anisotropic bending elasticity. Indeed, if the ribbon is composed of an isotropic material, the ratio of the elastic constants for bend in the easy and hard directions is $\epsilon_{e} / \epsilon_{h}=(t / w)^{2}[13]$. This strong dependence on the ratio of $t / w$ implies that the ribbon polymer shows a very anisotropic elastic response. On the chemical scale, the chains do not normally consist of an isotropic material, and details of chemical bonding are important for bend, thus, even higher ratios of $\epsilon_{e} / \epsilon_{h}$ are possible.

Some of the solution properties, in particular the liquidcrystalline behavior, have been studied theoretically [10]. Here we study the structure of a single ribbon polymer immersed in a poor solvent. Assuming the system is well be-

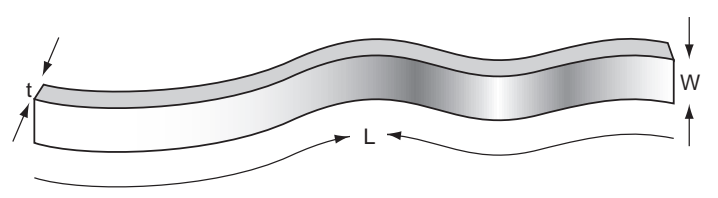

Fig. 1: Model for anisotropic semiflexible polymer

low the $\Theta$ temperature, the chain collapses completely and forms a condensate which is effectively a polymer melt.

For isotropic semiflexible chains such as DNA, the condensate often forms a toroid $[1,2,14-21]$ and sometimes a globule [22-25] through intermediate states [28-30]. For ribbon polymers, we show in this paper that the condensates forms an annulus. The annulus formation manages to avoid bend in the hard direction, while reducing contact of the chain with the solvent. The novel character of these annular condensates is that multilayer annuli can form and they undergo sudden changes as a function of chain length or surface tension. This is in marked contrast to the case of ordinary flexible or semiflexible polymers where no dramatic changes occur. This quantization is a direct consequence of the anisotropic elasticity.

To model the system, we introduce two surface tensions $\gamma_{h}$ and $\gamma_{e}$ which corresponds to the surface energies of contact between the top (narrow surface) of the polymer and 


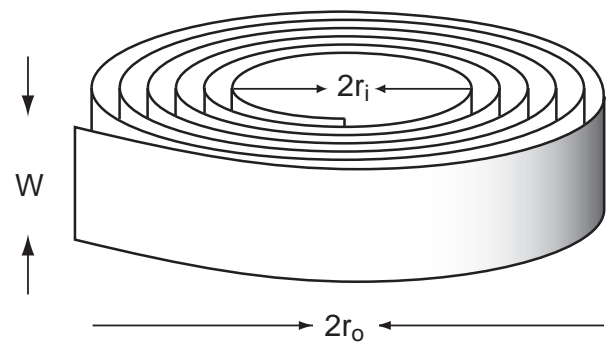

Fig. 2: Annular condensate for short ribbon polymer in poor solvent

the solvent, and the surface energies of contact between the side (flat and wide surface) of the polymer and the solvent, respectively. If the chain consists of anisotropic molecules, any ratio of $\gamma_{e} / \gamma_{h}$ is possible in principle. In this paper, however, we assume $\gamma_{e} / \gamma_{h} \approx 1$.

A large number of parameters appear in this model. Once final results have been obtained, it is useful to ignore numerical prefactors and to make some crude estimates. Then, we write $w \approx t \approx a$ and $\gamma_{e} \approx \gamma_{h} \approx g k T / a^{2}$, where $g$ is of order unity and $a$ is a length of order of one Angstrom. It is convenient to define two bare persistence lengths, $l_{e} \equiv$ $\epsilon_{e} / k T$ and $l_{h} \equiv \epsilon_{h} / k T$ which are essentially the scales on which a free chain bends in the two directions due to thermal fluctuations.

We begin by examining the shape of condensates when we slowly lengthen the chain. For short chains, bend costs elastic energy, then the chain more-or-less remains as a rod. For slightly longer chains, however, the overlap induced by bend compensates the elastic energy by reducing its surface energy, therefore, the chain may form a ring. For a circular ring of radius $R$ it is easy to show that the bending energy is $\frac{1}{2} \epsilon_{e} L / R^{2}$. The change in surface energy in bending into a ring is $4 \pi R w \gamma_{e}-2 L w \gamma_{e}$. Minimising it over $R$ and equating the free energies gives the critical length, $L^{*}=(3 \sqrt{3} \pi / 2) \sqrt{\epsilon_{e} /\left(\gamma_{e} w\right)}$ at which ring formation occurs. The chain overlaps by $1 / 2$ turn, at $L=L^{*}$. Our interest here is in the case $L \gg L^{*}$ so the chain winds around many times.

The simplest way the chain can pack is to form a disk with a hole in the middle (annulus) as shown in fig 2, This annulus has height $w$ and inner and outer radii $R_{i}$ and $R_{o}$, respectively. The total energy consists of three terms: (1) surface energy of the top and bottom surfaces, (2) surface energy of the inner and outer exposed sections of the annulus, (3) bending energy of the chain. This last term is due to bending only in the easy direction.

Because the volume of the chain is conserved, $R_{o}$ and $R_{i}$ are related by

$$
t L w=w \pi\left(R_{o}^{2}-R_{i}^{2}\right) .
$$

There is thus only one variable to minimize over (say $R_{i}$ ). Also, since the area of the upper and lower surfaces is fixed (i.e., both are always of area $t L$ ), there are really only two relevant terms in the total energy. The lateral surface energy is easily calculated as

$$
2 \pi \gamma_{e} w\left(R_{o}+R_{i}\right) .
$$

The bending energy is calculated by

$$
\frac{\epsilon_{e}}{2} \int_{0}^{L} d s\left(\frac{d \theta}{d s}\right)^{2}
$$

where $s$ is the arc length and $\theta$ is the angle between the tangent of the ribbon curve and some fixed direction in the plane of the disk.

In one complete turn of the ribbon, $\theta$ increases by $2 \pi$ and $s$ increases by $2 \pi r$ where $r$ is the distance of the chain from the center of the annulus. This gives $d \theta / d s=1 / r$. During the same change in $s$ the radius increases by one ribbon thickness, i.e., $d r / d s=t /(2 \pi r)$. This allows us to write the bending energy as $\pi \epsilon_{e} t^{-1} \ln \left(R_{o} / R_{i}\right)$. The total energy is then

$$
U=\frac{\pi \epsilon_{e}}{t} \ln \left(\frac{R_{o}}{R_{i}}\right)+2 \pi \gamma_{e} w\left(R_{o}+R_{i}\right),
$$

subject to $R_{o}^{2}=R_{i}^{2}+t L / \pi$.

It is convenient at this point to introduce two characteristic lengths. One is $\Lambda \equiv \epsilon_{e} /\left(2 \gamma_{e} w t\right)$, and the other is $\Gamma \equiv \sqrt{L t / \pi}$. For long chains, $\Gamma$ is much larger than $\Lambda$, and we shall see $R_{i} \approx \Lambda$ and $R_{o} \approx \Gamma$. In this limit, $\Lambda \ll \Gamma$, the annulus is "fat", i.e., it has a small hole in the middle. In the opposite limit, $\Lambda \gg \Gamma$, the hole is large and the annulus is "thin". It looks like a ring.

In the long-chain limit, $R_{i} \ll \Gamma$, the total energy is approximated by

$$
U=\text { const. }+2 \pi \gamma_{e} w R_{i}-\frac{\pi \epsilon_{e}}{t} \ln \left(\frac{\pi R_{i}}{\sqrt{L t}}\right),
$$

which has a minimum at $R_{i}^{*}=\Lambda$. Using our crude estimates give $R_{i}^{*} \approx l_{e} / g$, i.e., the internal radius is somewhat less than the bare persistence length. In this limit the internal radius of the annulus is independent of chain length, whereas the external radius is approximately $R_{o} \approx(t L / \pi)^{1 / 2}$. A more accurate expression for $R_{i}^{*}$ can be found by expanding in ascending powers of $\alpha \equiv \Lambda / \Gamma$. This gives $R_{i}^{*}=\Lambda\left(1-\alpha+\frac{3}{2} \alpha^{3}-\ldots\right)$. From this it is clear that, even though $R_{i}^{*}$ becomes asymptotically $\Lambda$, the approach is rather slow as $L^{-1 / 2}$.

In the limit $\Lambda \gg \Gamma$, we can again expand the total energy and find the minimum at $R_{i}^{*}=\left(\Gamma^{2} \Lambda / 2\right)^{1 / 3}$, or more crudely, $R_{i}^{*} \approx\left(L l_{e} a / g\right)^{1 / 3}$. The crossover between the thin and fat regimes occurs at $\Gamma=\Lambda$. A crude estimate of this gives $L_{c}=a\left(L^{*} / a\right)^{4}$. This implies that the chain must be rather long to be in the fat regime.

The results just derived correspond to the case of one layer. The novel point about this system is that it can choose to form more than one layer. This decreases the surface area exposed by the top and bottom surfaces but comes at the expense of extra surface energy on the inside and outside surfaces and extra bend both in the hard and 


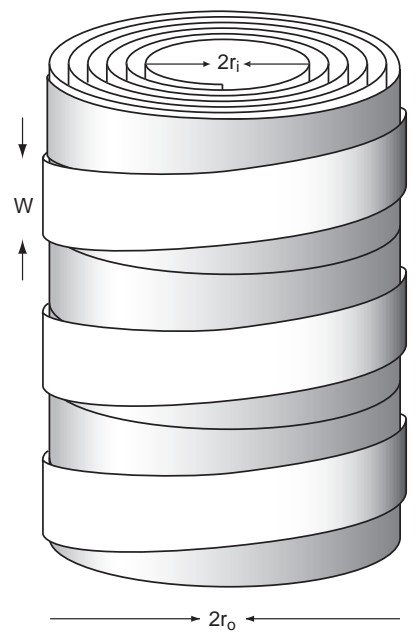

Fig. 3: tubular condensate for long ribbon polymer in poor solvent

easy directions. For second layer to form the chain must bend in the hard direction. The best way of doing this is to join the two layers together at the outer radius (fig 3 ).

We need to calculate the bending energy of the chain in the hard direction. The chain must bend between the two layers. It must jump a distance $w$ in the vertical direction while winding a distance $\Omega=2 \pi R_{o}$ in arc length. If we let $\phi$ be the angle made by the chain with the plane of the lower annulus then the bending energy can be written as

$$
U_{h}=\frac{\epsilon_{h}}{2} \int_{0}^{\Omega} d s\left(\frac{d \phi}{d s}\right)^{2} .
$$

This is to be minimized subject to a constraint that the chain jumps up a distance $w$, i.e., $\int_{0}^{\Omega} d s \phi=w$. Here we have made the very good approximation that the angle $\phi$ is always small. Using the method of Lagrange multipliers and the Euler-Lagrange equations we find that the minimum energy trajectory coresponds to the solution of the differential equation: $d^{2} \phi / d s^{2}=$ const. Using the boundary conditions $\phi(0)=0=\phi(\Omega)$ the constraint gives

$$
\phi(s)=\frac{6 w}{\Omega}\left\{\frac{s}{\Omega}-\left(\frac{s}{\Omega}\right)^{2}\right\},
$$

and the bending energy becomes

$$
U_{h}=\frac{6 \epsilon_{h} w^{2}}{\Omega^{3}} .
$$

It is fairly clear from this formula that the chain wants to spread the bend over as much length as possible, so that it is best to bend at the outer radius of the annulus and $\Omega=2 \pi R_{o}$.

We now examine the transition from one to two layers. The one-layer energy is

$$
U_{1}=\frac{\pi \epsilon_{e}}{t} \ln \left(\frac{R_{o}}{R_{i}}\right)+2 \pi \gamma_{e} w\left(R_{o}+R_{i}\right)+2 \gamma_{h} L t,
$$

subject to $R_{o}^{2}=R_{i}^{2}+t L / \pi$. The two-layer energy is

$$
\begin{aligned}
U_{2}= & \frac{2 \pi \epsilon_{e}}{t} \ln \left(\frac{R_{o}}{R_{i}}\right)+4 \pi \gamma_{e} w\left(R_{o}+R_{i}\right) \\
& +\gamma_{h} L t+\frac{6 \epsilon_{h} w^{2}}{\left(2 \pi R_{o}\right)^{3}},
\end{aligned}
$$

subject to $R_{o}^{2}=R_{i}^{2}+t L / 2 \pi$. Here $\gamma_{h} L t$ is the surface tension of the "top" of annulus.

The transition from one to two layers occurs when $U_{1}=$ $U_{2}$. To solve this equation we need to minimize $U_{1}$ over $R_{i}$ and similarly for $U_{2}$. In principle different $R_{i}$ will be associated with the two geometries. However, in the limit where $R_{i} \ll(L t)^{1 / 2}$ the result is the same for both, i.e., $R_{i}^{2} \approx \epsilon_{e} /\left(2 \gamma_{e} w t\right)$. This gives

$$
\begin{aligned}
U[n]= & \frac{n \pi \epsilon_{e}}{t}\left\{1+\ln \left(\frac{2 \gamma_{e} t w}{\epsilon_{e}} \sqrt{\frac{L t}{(n \pi)}}\right)\right\} \\
& +\frac{2}{n} \gamma_{h} L t+2 \gamma_{e} \pi w \sqrt{\frac{n L t}{\pi}} \\
& +\frac{3(n-1) \epsilon_{h} w^{2}}{\sqrt{2} \pi^{3}}\left(\frac{L t}{\pi}\right)^{-3 / 2},
\end{aligned}
$$

where $n$ is the number of layers (1 or 2). Equating the two energies for $n=1$ and $n=2$, and ignoring the logarithmic factors, gives

$$
\begin{aligned}
\frac{\pi \epsilon_{e}}{t}-\gamma_{h} L t+ & 2(\sqrt{2}-1) \pi w \gamma_{e} \sqrt{\frac{L t}{\pi}} \\
& +\frac{3 \epsilon_{h} w^{2}}{\sqrt{2} \pi^{3}}\left(\frac{L t}{\pi}\right)^{-3 / 2}=\quad 0 .
\end{aligned}
$$

The four terms in this equation represent the four kinds of energy differences between the two layer and one layer system. In order these are: Easy bend; top/bottom surfaces; side surfaces; hard bend. The driving force for the transition to two layers is the top/bottom surface energy. This is opposed by the other three terms. We are mainly interested here in the case where the hard bend is the dominant penalty. In this case the transition occurs when

$$
L^{5} \approx \frac{\epsilon_{h}^{2} w^{4}}{\left(\gamma_{h}^{2} t^{5}\right)}
$$

where we have ignored numerical prefactors. Even more approximately this can be written $L \approx a\left(l_{h} / a\right)^{2 / 5}$ where $l_{h}$ is the persistence length in the hard direction. Provided $l_{h} \gg l_{e}$ (it is true when $L \gg R_{i}$ ) our approximations are valid.

At the transition the jump in $R_{i}$ is small, since $R_{i}$ is about the same for both layers. However, there is a sudden jump in $R_{o}$ by a factor of $1 / \sqrt{2}$. In the case of a three layer system, the chain must jump once on the outside radius and once on the inside radius. Since the jump on the inside radius must take place over a shorter amount of 


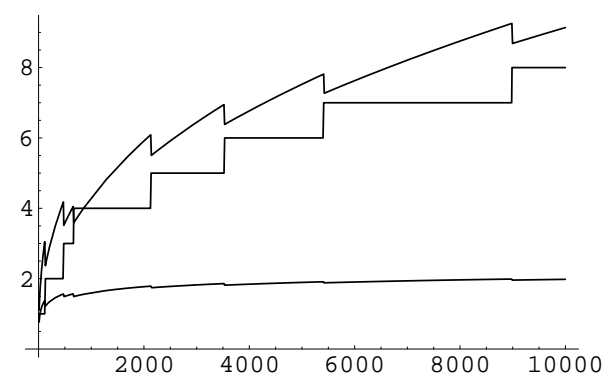

Fig. 4: Layering transition in a fat condition $\Gamma / \Lambda \approx 10$. The horizontal axis is the polymer length $L / w$. The upper and the lower curves represent $R_{o}$ and $R_{i}$ respectively and the middle step line represents the height of the tubular condensate in unit of ribbon width $w$.

arc length, its energy cost is expensive. The energy for $n$ layers is then

$$
\begin{aligned}
U[n]= & \frac{n \pi \epsilon_{e}}{t} \ln \left(\frac{R_{o}}{R_{i}}\right) \\
& +n\left(2 \pi \gamma_{e} w\right)\left(R_{o}+R_{i}\right)+\frac{2}{n} \gamma_{h} L t \\
& +\frac{6 \epsilon_{h} w^{2}}{(2 \pi)^{3}}\left(\left[\frac{n}{2}\right] R_{o}^{-3}+\left[\frac{n-1}{2}\right] R_{i}^{-3}\right),
\end{aligned}
$$

with $R_{o}=\left(R_{i}^{2}+n^{-1} t L / \pi\right)^{1 / 2}$, and where $[x]$ indicates the integer part of $x$.

In order to show the layering transition of equilibrium form in a fat condition, in fig 4 we plot the outer radius of the condesates $R_{o}$, the radius of the inner hole of the condensates $R_{i}$, and the quantized height of the condensate $n w$. This fat condition is always achieved in the long chain limit. For large $L$, the size of the condensate is roughly proportional to $\sqrt{L}$. Below the medium chain length, there exists a certain parameter region where the condensate is thin. We show in fig 5 behavior of the layering transition in a thin condition.

We have assumed here that the system can form a welldefined annulus. It is important and interesting to examine the fluctuations around the equilibrium shape of the system. We will concentrate here on the case of a single layer. There are two types of fluctuations. The first kind is fluctuations of the shape in the radial direction, i.e., distortion from a circle. We discuss it later.

The second kind is fluctuations out of the plane which cause two kinds of energy penalty. The first is arises from hard bend and the energy penalty is $\epsilon_{h} / 2 \int_{0}^{L} d s(d \phi / d s)^{2}$, where $\phi$ is the angle made by the tangent to the ribbon out of the plane of the annulus. The second arises from the surface area exposed due to differences in the height $z(s)$ of the layers. This gives rise to an energy penalty $\gamma_{e} \int_{2 \pi R_{1}}^{L} d s|z(s)-z\{s-2 \pi r(s)\}|$. Noting that $z(s)=\int_{0}^{s} d s^{\prime} \phi\left(s^{\prime}\right)$ and expanding for small gradients gives

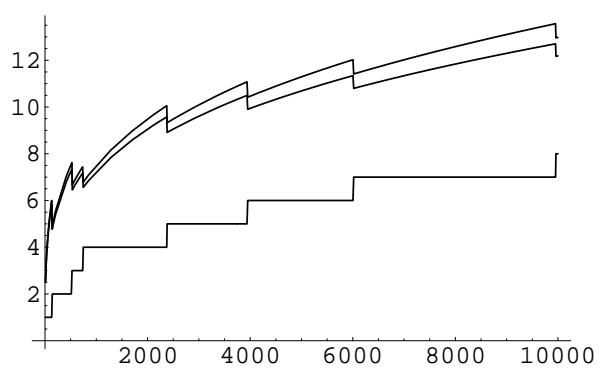

Fig. 5: Layering transition in a thin condition $\Gamma / \Lambda \approx 0.5$. The horizontal axis is the polymer length $L / w$. The upper two curves represent $R_{o}$ and $R_{i}$ respectively and the lower step line represents the height of the tubular condensate in unit of ribbon width $w$.

$2 \pi \gamma_{e} \int_{2 \pi R_{1}}^{L} d s|\phi(s)| r(s)$. The total energy penalty is then

$$
\frac{\epsilon_{h}}{2} \int_{0}^{L} d s\left(\frac{d \phi}{d s}\right)^{2}+2 \pi \gamma_{e} \int_{2 \pi R_{1}}^{L} d s r(s)|\phi(s)| .
$$

This kind of total energy is peculiar because of the form of the surface tension term, $|\phi(s)|$. This term is first order and not differentiable about the equilibrium position $\phi=$ 0 . This means that the usual eigenmode techniques we might have used to analyse the system are not applicable.

However, a good estimate of the size of the fluctuations and in particular $\delta z \equiv \sqrt{\left\langle(z(L)-z(0))^{2}\right\rangle}$ can be found by mapping the problem onto that of a semiflexible chain in a nematic field. From now on we ignore all numerical prefactors. For our purposes it is convenient to use an approach developed by Odijk [31,32]. He considers a wormlike chain directed by a parabolic potential $\mu \phi^{2}$, where $\mu$ is a constant. The chain has a typical angular deviation $\delta \phi \equiv \sqrt{\left\langle\phi^{2}\right\rangle}$. Such a chain can be viewed as a series of steps of size $\lambda_{\|}=l_{h}(\delta \phi)^{2}$ in the plane of the annulus and $\lambda_{z}=\lambda_{\|} \delta \phi=l_{h}(\delta \phi)^{3}$ perpendicular to the annulus. A chain of length $L$ undergoes an anisotropic random walk of $L / \lambda_{\|}$steps in the plane, with each step travelling a distance $\lambda_{z}$ perpendicular to the plane. This implies the deviation out of the plane is $\delta z=\sqrt{L l_{h}}(\delta \phi)^{2}$.

Odijk shows that the strength of the potential and the angular deviations are related by $(\delta \phi)^{4}=k T /\left(\mu l_{h}\right)$, so that $\delta z=\sqrt{k T L / \mu}$. This result cannot be applied directly to our problem, since we have a confining potential of the form $\gamma_{e} r(s)|\phi(s)|$. However, the details of the shape of the potential cannot matter for the gross behaviour of the system. In particular we can replace our potential by a harmonic one, provided we choose $\mu$ self-consistently, i.e., we choose $\mu$ so that for a typical value of the angular deviation the two potentials coincide. Equating the two potentials gives $\gamma_{e} r(s)|\phi|=\mu \phi^{2}$. This together, with the relation $(\delta \phi)^{4}=k T /\left(\mu l_{h}\right)$ gives $\mu=\left(\gamma_{e} r(s)\right)^{4 / 3}\left(l_{h} / k T\right)^{1 / 3}$. Note that since $r$ varies along the chain, the effective confining potential $\mu$ also varies along the chain. We can now integrate over the whole annulus to get the variation in height $\delta z$. Between $r$ and $r+d r$ there is a total length 
$r d r / t$ and hence the square of the step in the $z$ direction is $d z^{2}=\left(k T /\left(\gamma_{e} r\right)\right)^{4 / 3} l_{h}^{-1 / 3} t^{-1} r d r$. Integrating over the whole annulus gives:

$$
\delta z=\frac{k T}{\left(\gamma_{e} l_{h}\right)^{2 / 3}} \sqrt{\frac{l_{h}}{t}} \sqrt{R_{o}^{2 / 3}-R_{i}^{2 / 3}}
$$

Note that this deviation grows only very slowly with the chain length. In, particular, for very long chains we have $\delta z=\left(k T /\left(\gamma_{e} l_{h}\right)\right)^{2 / 3}\left(l_{h} / t\right)^{1 / 2} t^{1 / 6} L^{1 / 6}$. Setting $t=a$ and $\gamma_{e}=g k T / a^{2}$ gives $\delta z \approx a g^{-2 / 3}\left(L / l_{h}\right)^{1 / 6}$. Thus, in general the overall fluctuations will be less than, or of the same order as the annular thickness, $w$, and the annulus will be well-defined and relatively flat. This is the conclusion for one layer.

For many layers this conclusion remains true, since in that case the effective persistence length scales with the number of layers and the radius also scales with the number of layers. This implies that the absolute deviation in thickness remains roughly constant and the relative variation decreases as more layers are added.

We now discuss fluctuations of radial direction. The simplest mode is one where the inner radius changes from its equilibrium value, $R_{i}^{*}$. In the long-chain limit, where $R_{i}^{*} \ll \sqrt{L t}$ the energy penalty for deviating from equilibrium is

$$
\delta U=\frac{\pi \epsilon_{e}}{2 t}\left(\frac{R_{i}-R_{i}^{*}}{\Lambda}\right)^{2}
$$

Equating this to $k T / 2$ gives a typical size of fluctuation $\sqrt{\left\langle\left(R_{i}-R_{i}^{*}\right)^{2}\right\rangle}=\Lambda \sqrt{t /\left(\pi l_{e}\right)}$. For $l_{e} / t=10$ this gives a relative fluctuation of $13 \%$. We expect that the fluctuations within the plane will be reasonably small.

In conclusion, ribbon polymers which have large bending rigidities in lateral direction spontaneously form anisotropic condensates in poor solvents. The size of those peculiar condensates quantized as a variation of the length of polymers and solvent quality.

When the material is homogeneous, the surface tensions and elastic energies are determined by geometry of the ribbon. In general, for homogeneous materials, we may assume $\epsilon_{e}<\epsilon_{h}$ and $\gamma_{e} \approx \gamma_{h}$, which is the case we discussed in this paper. On the chemical scale, however, other anisotropic conditions are possible in principle. The ribbon polymers with $\epsilon_{e}<\epsilon_{h}$ and $w \gamma_{e}<t \gamma_{h}$ [33], form a different type of anisotropic condensates in poor solvents, that will be discussed somewhere else.

$$
* * *
$$

The authors thank Jean-Louis Sikorav for discussions and comments on the manuscript. This work was initiated with support by NTT Basic Research Laboratories. Y.Y.S. wishes to acknowledge CEA, IPhT and Takushoku University, RISE for financial support.

\section{REFERENCES}

[1] Klimenko S. M., Tikkchonenko T. I. and Andreev V. M., J. Mol. Biol., 23 (1967) 523

[2] Grosberg A. Yu., Biophysics, 24 (1979) 30

[3] Frank-Kamenetskit M. D., Anshelevich V. V. and Lukashin A. V., Sov. Phys. Usp., 30 (1987) 317

[4] Marko J. F. and Siggia E. D., Macromolecules, 27 (1994) 981

[5] OdiJk T., J. Chem. Phys., 105 (1996) 1270

[6] Marko J. F., Phys. Rev. E, 55 (1997) 1758

[7] Haijun Z., Yang Z. and Zhong-Can O.-Y., Phys. Rev. Lett., 82 (1999) 4560

[8] Schlüter A.-D., Adv. Mater., 3 (1991) 282

[9] Nyrkova I. A., Semenov A. N., JoAnny J.-F. and Khokhlov A. R., J. Phys. II (Paris), 6 (1996) 1411

[10] Nyrkova I. A., Semenov A. N. and Joanny J.-F., J. Phys. II (Paris), 7 (1997) 825

[11] Nyrkova I. A., Semenov A. N. and Joanny J.-F., J. Phys. II (Paris), 7 (1997) 847

[12] Golestanian R. and Liverpool T. B., Phys. Rev. E, $62(2000) 5488$

[13] Landau L. D. and Lifshitz E. M., Theory of Elasticity (Pergamon, London) 1970, p. 77

[14] Bloomfield V. A., Biopolymers, 31 (1991) 1471

[15] Bloomfield V. A., Biopolymers, 44 (1997) 269

[16] Li A.-Z., FAn T-Y. and Ding M., Science in China B, 35 (1992) 169

[17] Ubbink J. and Odijk T., Biophys. J., 68 (1995) 54

[18] Hud T., Downing K. H. and Balhorn R., Proc. Natl. Acad. Sci. USA, 92 (1995) 3581

[19] Park S. Y., Harries D. and Gelbart W. M., Biophys. J., 75 (1998) 714

[20] Pereira G. G. and Williams D. R. M., Biophys. J., 80 (2001) 161

[21] TAKenaka Y., Yoshikawa K., Yoshikawa Y., Koyama Y. and Kanbe T., J. Chem. Phys., 123 (2005) 014902

[22] Grosberg A. Yu. and Zhestkov A. V., J. Biomol. Struct. Dyn., 3 (1986) 859

[23] Vasilevskaya V. V., Khokhlov A. R., Kidoaki S. and Yoshikawa K., Biopolymers, 41 (1997) 51

[24] Jary, D. and Sikorav J.-L., Biochem., 38 (1999) 3223

[25] OdiJk T., Phil. Trans. R. Soc. Lond. A, 362 (2004) 1497

[26] Cooke I. R. and Williams D. R. M., Physica A, 339 (2004) 45

[27] Yoshikawa Y.,Yoshikawa K. and Kanbe T., Biophys. Chem., 61 (1996) 93

[28] Schnurr B., MacKintosh F. C. and Williams D. R. M., Europhys. Lett., 51 (2000) 279

[29] Pereira G. G. and Williams D. R. M., ANZiAM J., 45 (2004) C163

[30] Yoshinaga N., Yoshikawa K. and Онta T., Euro. Phys. J. E, 17 (2005) 485

[31] Odisk T., Macromolecules, 16 (1983) 1340

[32] Odisk T., Macromolecules, 19 (1986) 2313

[33] Nelson J. C., Saven J. G., Moore J. S. and Wolynes P. G., Science, 277 (1997) 1793 